\title{
Preparation, Properties, and Cell Attachment/ Growth Behavior of Chitosan/Acellular Derm Matrix Composite Materials
}

\author{
Tao Lu', Ruixin $\mathrm{Li}^{2}$, Yan Zhang ${ }^{2}$, Yuxian Yan', Yong Guo ${ }^{2}$, Jing Guan², Jimin $\mathrm{Wu}^{2}$, Zhihong Li ${ }^{2}$, \\ Bo Ning ${ }^{2}$, Shujie Huang ${ }^{2}$, Xizheng Zhang, ${ }^{2, *}$
}

\author{
${ }^{1}$ Affiliated Hospital of Chinese People's Armed Police Force Medical College, Tianjin, China; ${ }^{2}$ Institute of Medical Equipment, \\ Tianjin, China. \\ Email: z84656716@yahoo.com
}

Received December $3^{\text {rd }}, 2010$; revised January $1^{\text {st }}, 2011$; accepted January $9^{\text {th }}, 2011$.

\begin{abstract}
Composite membranes and sponge scaffolds consisting chitosan (CS) and acellular derm matrix (ADM) in six ratios were prepared by solvent evaporation technique and freeze-drying method, respectively. The composite materials were characterized by water contact angle measurement, scanning electron microscopy (SEM), water absorption and HaCat cells compatibility. The SEM result showed that CS/ADM three-dimensional (3D) micro-porous structures were successfully produced. The water absorption value of all scaffolds was over 18 times of its initial weight, which is high enough for skin regeneration scaffold, but there were no significant differences of water absorption ratio between deionized water and PBS solution for same scaffold $(P>0.05)$. HaCat cells were distributed uniformly on the surfaces of membrane 4 - 6, and an almost confluent monolayer was formed on membrane 6 on the fifth day, whereas cells maintained round and spherical in shape on the surface of membrane 1. The results showed that the cell compatibility of pure CS membrane needed to be improved, and addition of ADM realized this purpose. The results of compatibility of HaCat cells on scaffolds showed that the cell proliferated well on the scaffolds 3 and 4. In our study, the cell's attachment and growth on the composite membranes was mainly determined by the content of the membrane, whereas the cell's attachment and growth in the scaffolds was determined by both the content and structure of the scaffolds.
\end{abstract}

Keywords: Chitosan, Acellular Derm Matrix, Membrane, Scaffolds, HaCat Cell Compatibility

\section{Introduction}

Skin being the largest and most highly complex organ in the human body is the most affected organ in injuries [1]. Every thing is done to reduce risks for health, especially from the growing number of synthetic compounds and new formulations [2]. Skin corrosivity testing in vivo may cause severe discomfort and pain to test animals. Therefore, many attempts have been made to replace the in vivo test in laboratory animals. Skin corrosion is defined as the production of irreversible tissue damage in the skin following the application of a test material (OECD, 2002) [3]. The new OECD Test Guideline 431 "In Vitro Skin Corrosion" (OECD, 2004) [4] defines the requirements for in vitro skin models to be validated for skin corrosivity testing and defines general and functional model conditions that need to be evaluated before the skin models will be routinely used. The most impor- tant general conditions are a multi-layered, functional stratum corneum with the necessary lipid profile, and absence of any contamination. The most important functional conditions specified in TG 431 are a stable and sufficiently high cell viability (expressed as metabolic conversion capacity), a sufficient resistance to a slowly penetrating cytotoxic marker chemical, reproducibility of data over time and between laboratories, and finally, capability to correctly classify twelve reference chemicals specified in TG 431. But in China, only a few works have been done about it, so we try to reconstruct a new human epidermal model scaffold.

Chitosan (CS) is an abundant, naturally occurring polysaccharide obtained by deacetylation of natural chitin [5]. It is biocompatible, biodegradable, easily formed into structures under mild processing conditions and can be chemically modified, so it is a natural choice as drugdelivery carrier [6,7], cartilage/skin tissue engineering 
scaffolds [8-10], and regenerative membrane [11]. The physical properties of a polymer can be altered by introducing a second polymer that improves the properties of the original polymer in certain aspects, such as hydrophobility, cell compatibility. CS and some of its complexes have been studied for a number of biomedical applications, including wound dressings, drug delivery systems and space-filling implants [12-18]. In a study comparing purified collagen, naturally occurring extracellular matrix (ECM) scaffolds, and synthetic scaffold materials for in vitro endothelial cell attachment [19], it was found that ECM possessed the ability to recruit circulating marrow-derived progenitor cells and attract mature endothelial cells from selected organs such as the heart and liver to promote successful vascularization of engineered tissue structures. These studies reveal that extracellular components in a cell-free or acellular derm matrix (ADM) are critical for success in biomedical applications as scaffolds. HaCat cells, a human keratinocyte line, are commonly utilized as an in vitro cell model for toxicity testing and the discernment of process of chemically induced skin carcinogenesis.

In this study, we prepared CS/ADM composite membranes and scaffolds by solvent evaporation technique and freeze-drying method, respectively, and investigated the characteristics of composite materials by water contact angle measurement, scanning electron microscopy (SEM) observation, water absorption ratio and HaCat cells compatibility test.

\section{Methods}

\subsection{Materials}

$\mathrm{CS}(\mathrm{Mw} \approx 20000$, degree of deacetylation of $75 \%-85 \%)$ was purchased from the Sigma Chemical Company (Sigma-Aldrich Co.Ltd., USA) and used without further purification. Dulbecco's modified eagle's medium (DMEM), fetal calf serum (FCS) and trypsin-EDTA $(1 \times)$ were purchased from Gibco Laboratories (Invitrogen Corporation, CA, USA). All other chemicals were of analytical grade and used without further purification.

\subsection{Preparation of CS/ADM Composite Membranes and Scaffolds}

ADM was prepared as follows: Porcine skin of $0.3-0.4$ $\mathrm{mm}$ in thickness was obtained by removing the epidermal. It was digested in $0.25 \%$ trypsin solution at $37^{\circ} \mathrm{C}$ for $24 \mathrm{~h}$ to remove the epidermis and other cellular components. The remaining dermis was then immersed in $0.5 \%$ Triton X-100 solution for $36 \mathrm{~h}$ with continuous shaking to further remove cellular components, and subsequently washed with deionized water to obtain ADM. $\mathrm{ADM}$ particles was prepared by milling the prepared ADM.
Acetic acid was used as a solvent. To avoid the ADM particles aggregation, an alternative method was developed. First, ADM particles were scattered in water by stirring, and then CS powder, not solution, was added with strong stirring to ensure that the powder was uniformly mix with ADM particles. Finally, acetic acid was added to the solution. Since the CS powder was already uniformly dispersed, the addition of acetic acid caused the CS powder to immediately dissolve, thus avoiding the aggregation caused by ADM particles. Therefore, a homogeneous solution was obtained. The proportions of CS powder and ADM particles are listed in Tab.1. The resulting solution was allowed to stand at $4^{\circ} \mathrm{C}$ until all air bubbles had disappeared.

Composite membranes were prepared by solvent evaporation technique as follows: the mixture solution was cast into cell culture plates and allowed to dry at ambient temperature to form the composite membranes. The prepared composite membranes were neutralized with 1 wt.\% aqueous $\mathrm{NaOH}$ solution for $30 \mathrm{~min}$ and subsequently washed with deionized water to remove the remaining $\mathrm{NaOH}$. Composite porous scaffolds were prepared by using the same mix solutions: the mixture solution was cast into cell culture plates and frozen at $-20^{\circ} \mathrm{C}$, and the composite scaffolds were prepared by freezedrying method. The prepared composite scaffolds were neutralized with 1 wt.\% aqueous $\mathrm{NaOH}$ solution for $2 \mathrm{~h}$ and subsequently washed with deionized water to remove the remaining $\mathrm{NaOH}$. All the membranes and scaffolds were sterilized before seeding cells.

\subsection{Water Contact Angles and Wettability of Composite Membranes}

The water contact angles of the surface of the composite membranes were measured with HARKE SPCA contact angle goniometer (Beijing Harke Instrument Company). Distilled water was deposited on the surface of the samples with an automatic pipette. After being deposited, all contact angle measurements were taken within $20 \mathrm{~s}$. With a digital camera a computer image of the drop was determined, and the water contact angle $\theta$ was calculated according this formula: $\operatorname{tg}(\theta / 2)=h / r$, where $h$ is the high of the water drop and $r$ is the contact radius between water drop and the bottom. Figure 1 shows the schematic diagram of water contact angle. All measurements were

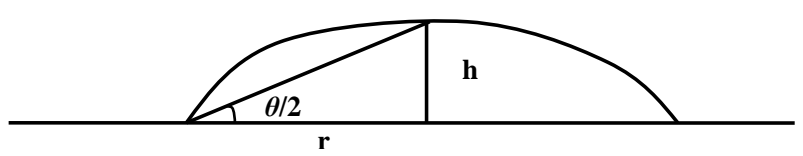

Figure 1. Schematic diagram of water contact angle. 
taken at an ambient temperature $\left(25^{\circ} \mathrm{C}\right)$ and they were repeated four times for each sample.

\subsection{H.E staining of Sponge Scaffold}

Scaffold samples were first dehydrated with an increasing series of alcohol concentrations $(30 \%, 50 \%, 70 \%$, $90 \%, 100 \%)$ and then embedded in paraffin. Paraffinembedded samples were sectioned at a thickness of $5 \mathrm{~mm}$. After removing the paraffin, samples were stained with hematoxylin and eosin. After sealing, samples were examined by light microscopy to inspect the degree of acelluar.

\subsection{SEM Observation}

For SEM observation, the specimens were fixed with $1.5 \%$ glutaraldehyde in $0.14 \mathrm{~m}$ sodium cacodylate buffer (pH 7.3), then dehydrated in graded alcohols, criticalpoint dried, sputter-coated with gold and analyzed in a SEM equipped (HITACHI S-3400N) at an accelerating voltage of $30 \mathrm{KV}$ and current of $119 \mu \mathrm{A}$.

\subsection{Water Absorption Ratio of Sponge Scaffold}

The CS/ADM composite scaffolds were incubated in deionized water and PBS solution at $37^{\circ} \mathrm{C}$ for $24 \mathrm{~h}$, and swelling continued to reach constant weight of the sample. Before weighing the sample, surface water was removed with filter paper. The water absorption ratio (R) was calculated by the following equation:

$$
\mathrm{R}=(\mathrm{Ws}-\mathrm{Wi}) / \mathrm{Wi}
$$

where Ws is the weights of swollen state and Wi is the weight of initial sample (before being immersed in water or PBS).

Each value was averaged from three parallel measurements.

\subsection{Cell Compatibility}

HaCat cells, an immortalized but non-tumorigenic human epidermal keratinocyte cell-line which retain their differentiation potential [20], were routinely cultured in high glucose DMEM medium supplemented with $10 \%$ (v/v) fetal calf serum, $10 \mu \mathrm{g} / \mathrm{ml}$ of streptomycin and 10 $\mathrm{U} / \mathrm{ml}$ of penicillin, and incubated at $37^{\circ} \mathrm{C}$ in a $5 \% \mathrm{CO}_{2}$, 95\% air humidified atmosphere.

\subsubsection{Cell Compatibility of Membranes}

HaCat cells were detached from the culture plated with $0.25 \%$ Trypsin, centrifuged, and then suspended in medium. The cell concentration was determined by manual count with a hemocytometer. $350 \mu \mathrm{l}$ cells suspension at density of $12 \times 10^{4} / \mathrm{ml}$ was seeded to each well (6-well culture plate) with composite membranes covered bottom and four wells for each sample. Cell proliferation was measured at 1, 3, 5, 7 days using the 3-(4, 5-dimethylthiazol-2-yl)-2, 5-diphenyl tetrazolium bromide (MTT) assay (Tianjin Runtai Reagent Company). Each sample was measured at 8 times. H.E staining also applied to the cultural plates with cells.

\subsubsection{Cell Compatibility of Scaffolds}

Scaffolds were sliced into $(0.0040 \pm 0.0001)$ gram pieces, and sterilized before use. $100 \mu 1$ cells suspension at density of $10 \times 10^{4} / \mathrm{ml}$ were seeded to each small prewetted scaffold, and four scaffolds for each kind of sample. Cell proliferation was measured at 1, 3, 5, 7 days using the 3-(4, 5-dimethylthiazol-2-yl)-2, 5-diphenyl tetrazolium bromide (MTT) assay in TECAN Elisa Reader.

\subsection{Statistical Analysis}

Differences between groups were analyzed by the SARS (version 11.0, http://www.pinggu.org/bbs/a-385796.html). Differences were considered to be significant at $P<0.05$.

\section{Results and Discussion}

\subsection{Water Contact Angles of CS/ADM Composite Membranes}

The material-cell interaction is affected by many factors, such as wettability (hydrophilicity/hydrophobility), surface free energy, chemistry, charge, roughness, and rigidity. The surface properties of scaffold are critical for its application because the surface of the scaffold is the place where the material interacts with the bioenvironment and where the cells attach and proliferate. Hydrophilicity is an important characteristic property for biomaterials. To determine the hydrophilicity of the membranes, their contact angles were investigated.

The higher contact angle indicates lower hydrophilicity. The water contact angles on the membranes are shown in Figure 2. As a consequence, the contact angle

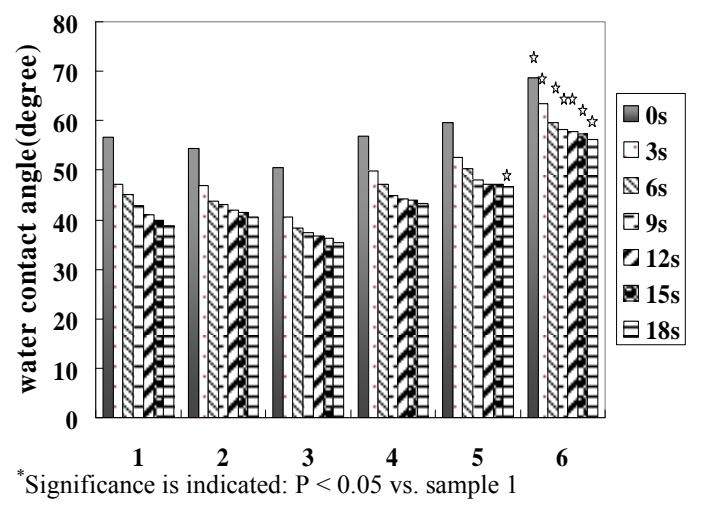

Figure 2. Water contact angle of composite membranes $(\mathbf{n}=$ 4). 
of all membranes decreased from 0 s to $18 \mathrm{~s}$. In addition, the contact angle of membrane 1 to 5 exhibited no significant difference, and membrane 6 exhibited the highest contact angle, which was primarily due to the surface become rougher with small ADM fibers scattered in the CS. Although the wettability of membrane was changed by blending with $\mathrm{ADM}$, the contact angles for all the materials were less than $70^{\circ}$, indicating that all these materials had good hydrophilicity.

Additionally, by comparing the data at different measuring times, we found that the contact angles decreased with time, indicating that the hydrophilicity of the matrices increased with the absorption of water.

The contact angle of membrane has been considered to be one of the physical parameters which related the affinity between cells and the matrix membranes. However, no correlation between the initial cell anchoring rate and the wet contact angle was found for the HaCat cells in the present study.

\subsection{H.E Staining of Scaffold}

H.E staining photograph of scaffold 4 is shown in Figure 3 , in which whole cells are absent from the scaffolds. This indicated the preparation of acellular derm matrix was successful.

\subsection{SEM Observation}

$\mathrm{CS}$ is a crystalline polysaccharide and is normally insoluble in aqueous solutions above $\mathrm{pH} 7$. However, in dilute acids $(\mathrm{pH}<6)$, the free amino groups are protonated and the molecule becomes soluble. This pH-dependent solubility provides a convenient mechanism for processing under mild conditions [21]. Figure 4 shows the SEM image of scaffold 1, 4 and 5. This image revealed the freeze-drying process generated an open pore microstructure with a high degree of interconnectivity. But with the increasing of ADM content, the pore uniformity decreased. Pure chitosan scaffold had the best pore structure.

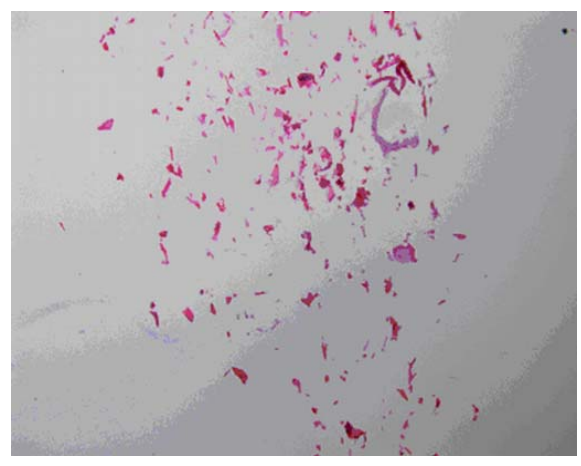

Figure 3. H.E staining of scaffold $4(\times 100)$.
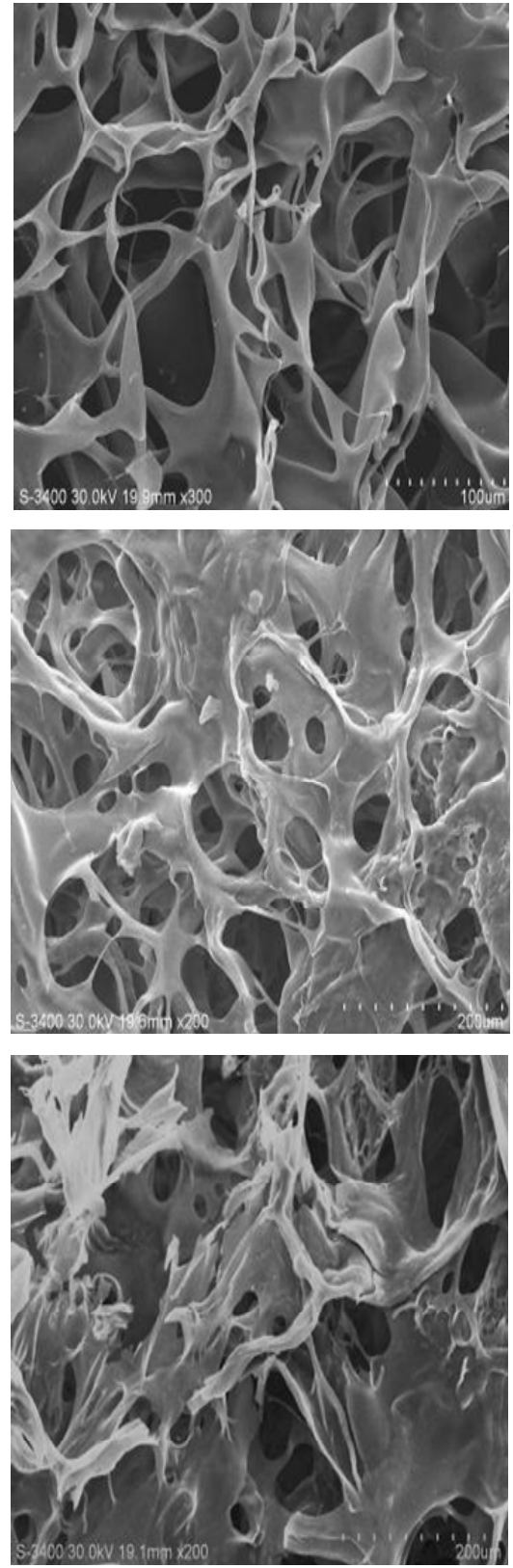

Figure 4. SEM image of scaffold 1, 4, 5.

\subsection{Water Absorption Ratio of Composite Scaffolds}

The ability of a scaffold to preserve water is an important property for skin regeneration. The water absorption ratios of various scaffolds are shown in Table 2. The water absorption ability of the CS/ADM scaffold could be attributed to both of their hydrophilicity and the maintenance of their three-dimensional structure. Differences between sample 1 and other groups were analyzed by the SARS and the result was $P<0.05$. So, there were sig- 
nificant differences of water absorption ratio between pure CS scaffold and composite scaffolds. The main reason was the pore structure of pure CS was the best, which could be proved by SEM images in Figure 4. In addition, differences between deionized water and PBS solution groups were analyzed by the SARS and the result was $P>0.05$ for each sample. So, there were no significant differences of water absorption ratio between deionized water and PBS solution for same scaffold.

The scaffold provides a necessary template and physical support to guide the differentiation and proliferation of cells into targeted functional tissues or organs. The scaffold should contain its three-dimensional structures in liquid culture medium. It should absorb body fluid for transfer of cell nutrients and metabolites through the material [22]. Transport issues such as nutrient delivery, waste removal, protein transport, gaseous exchange, and general vascularization and guided tissue regeneration are governed by the pore structure of the scaffold [23]. In our study, the absolute water absorption value of all scaffolds was over 18 times of its initial weight, which is high enough for skin regeneration scaffold. This result is similar with other studies on CS scaffolds. Ma et al. [22] fabricated freeze-dried CS-collagen scaffolds that were crosslinked with glutaric dialdehyde (GTA). They found that crosslinking decreased the swelling ratio from 16 to 8.

\subsection{Morphology of HaCat Cell}

Figure 5 shows the morphology of HaCat cells on culture plate photographed by a phase contrast microscope. Cells exhibited flagstone shape and form a confluent after being cultured for 3 days.

\subsection{Morphology and Proliferation of HaCat Cell on the Surface of Composite Membranes}

\subsubsection{Morphology of HaCat Cell Adhered on the Surface of Composite Membranes}

Prepared pure CS membrane was transparent, whereas composite membranes were opaque with white small fibers scattered in the membranes. In our study, the prepared membranes stick to the culture plates tightly, so that the cells could adhere on the surface of the membrane instead of on the culture plates.

The cytocompatibility of the matrices is very important for their applications. Since it is the biomaterial surface that first comes into contact with the living tissue when the biomaterial is planted in the body, the initial response of the body to the biomaterial depends on its surface properties. Surface properties that can influence biocompatibility include surface charge, surface topography, etc [24-29].

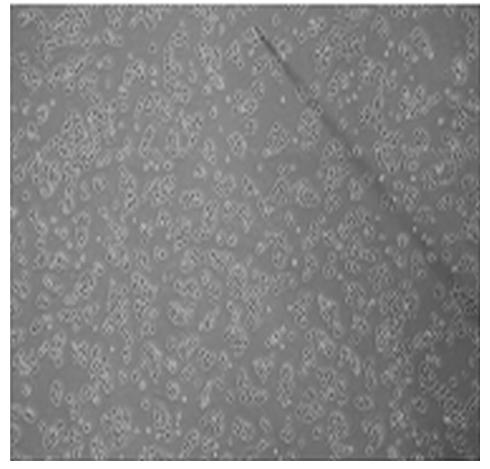

$1 \mathrm{~d} \times 40$

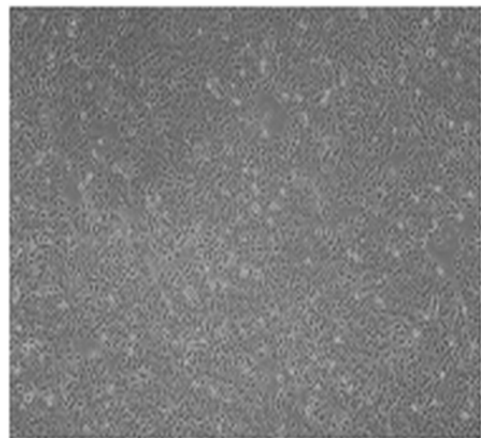

$3 \mathrm{~d} \times 40$

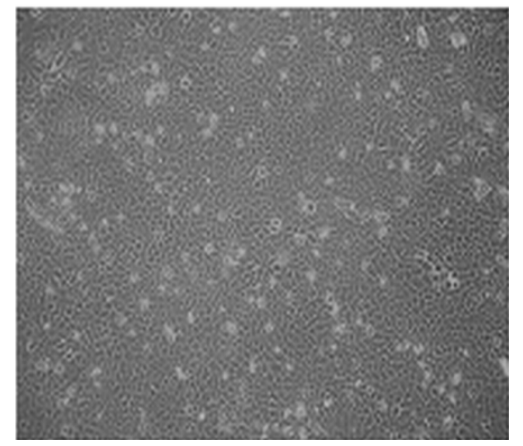

$3 \mathrm{~d} \times 100$

Figure 5. Phase contrast images of HaCat cell on culture plates.

To examine the cell-matrix interactions, HaCat cells were cultivated on the surface of the composite membranes, their morphology and distribution were observed and photographed by a phase contrast microscope on day 1, 3, 5, 7 d. Figure 6 and Figure 7 show the morphology of HaCat cell on the composite membrane on day 1 and day 7 , respectively. The micrographs of a 1-day culture reflect the status of HaCat cell attachment and spreading. Figure 6 shows that the attached cells on membrane 1 (pure CS membrane) were round and spherical in shape. Whereas the cells on the other membranes were flat, polygonal, and the difference of membrane 2 to 6 in spreading was not distinct. Chen et al. [30] concluded that cells 
with a flat shape survive better than cells with a more rounded shape. This result revealed that introduction of ADM to the CS membrane surfaces increased the number of cells attached on day 1 . This means that most cells had finished attachment and were in the process of spreading. Since spreading is an essential step in cell adhesion prior to exponential growth phase [31], a greater extent of cell spreading can have a profound elect on cell adhesion and growth. The distributions of HaCat cells cultured for 7 days are shown in Figure 7. It can be seen that the viable HaCat cells on composite mem- branes was much more than on CS membrane, indicating that the composite membranes could accelerate the differentiation of HaCat cells. It is known that the surface morphology of the substrate can exhibit a significant influence on the attachment, proliferation and function of cells in addition to the surface chemistry [32]. The surface of pure CS membrane showed a smooth photography. After addition of ADM, the roughness of the composite membrane surface increased. From Figure 62 to 6 it can be observed that the small ADM fibers scattered in the CS.
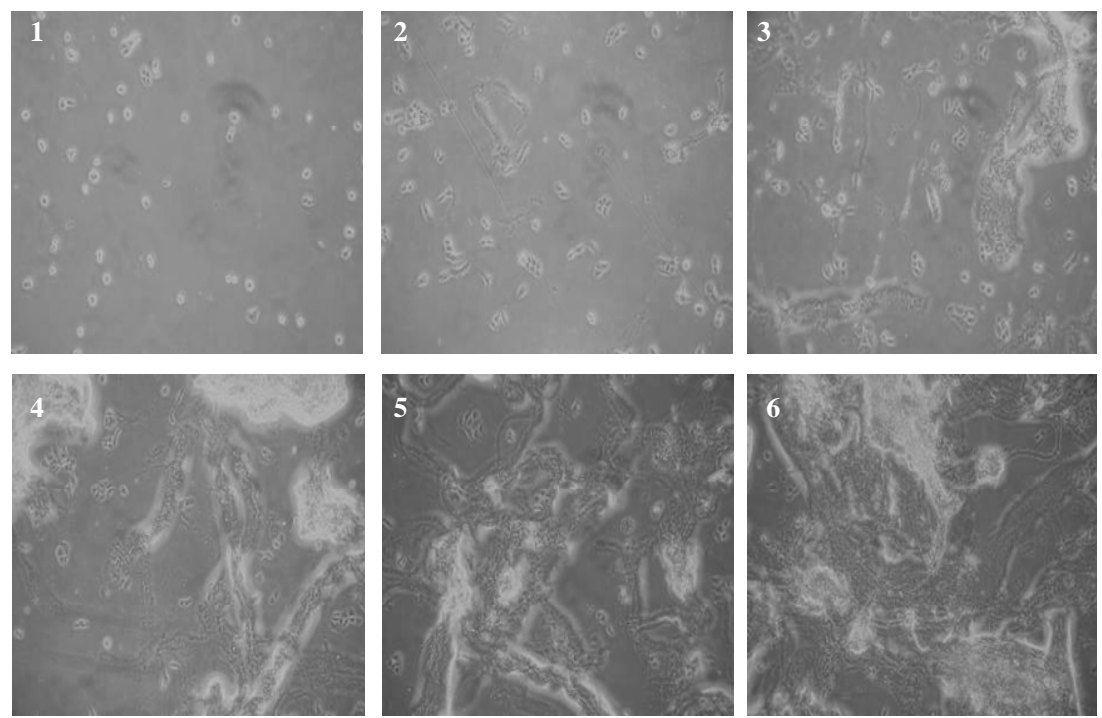

Figure 6. Phase contrast images of HaCat cells on the composite membranes on day $1(\times 100)$.
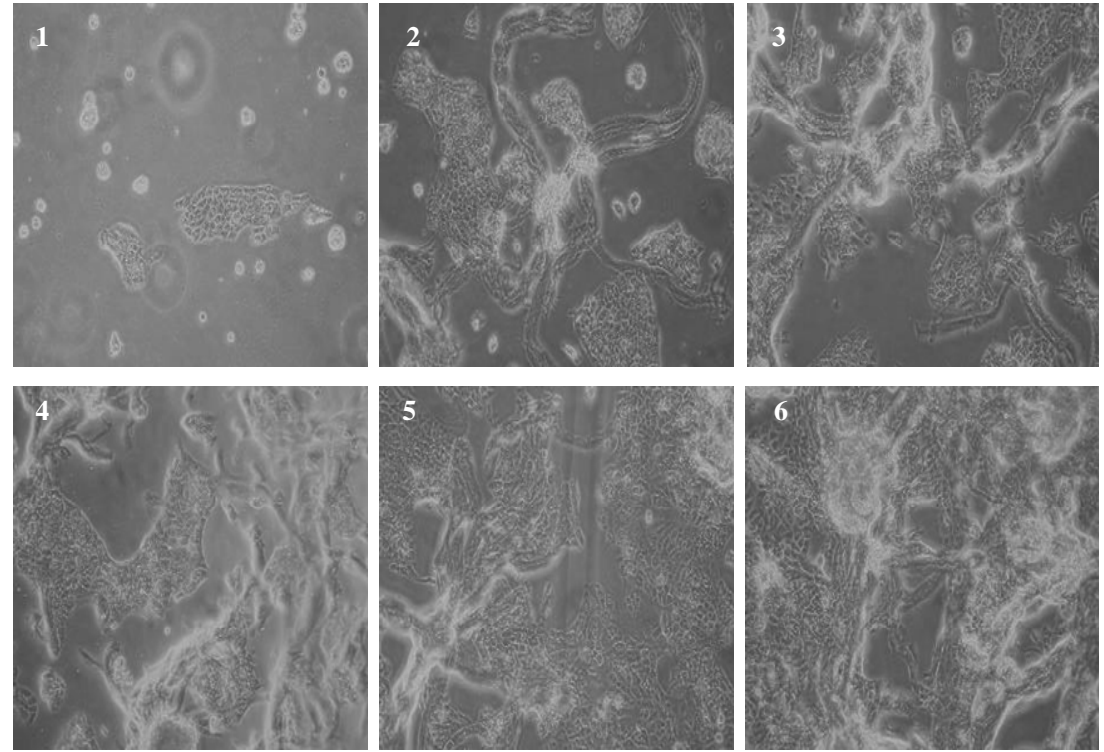

Figure 7. Phase contrast images of HaCat cells on the composite membranes on day $7(\times 100)$. 
In order to visualize cell morphologies on different membrane surfaces, cells and membranes were stained using Hematoxylin and Eosin Staining Kit (H.E) and observed with a microscope (BX51, Olympus, Japan). Figure 8 shows a series of images of HaCat cells adhered onto the membranes which was stained red. HaCat cells were distributed uniformly on the surfaces of membrane 4-6, and an almost confluent monolayer was formed on membrane 6 on the fifth day, whereas cells maintained round and spherical in shape on the surface of membrane 1 . The results showed that the cell compatibility of pure CS membrane needed to be improved, and addition of ADM realized this purpose.

\subsubsection{Proliferation of HaCat Cells on the Surface of Composite Membrane}

MTT assay was used as a measure of relative cell viability. HaCat cells were cultured on the membranes for 1,3 , $5,7 \mathrm{~d}$, and cell proliferation was determined by the MTT method. Figure 9 shows the viability of HaCat cell on different surfaces with different cultural time. There was significant enhancement of cell proliferation and viability on the composite membranes compared with that on pure CS membrane $(p<0.05)$. Also, cells proliferation rate on membrane 6 was the highest, the reason perhaps was that the content of ADM was similar to the ECM environment in vivo. In addition, cell adhesion on the surfaces increased and then decreased with a prolonged time period of culture. The cells formed a confluent monolayer on day 5 , and their proliferation were inhibited in the following day resulted in a decrease of viability. From the results of cell culture, we can conclude that composite film is bioactive to HaCat cells by increasing the attachment, spreading and proliferation of the cells. This trend was not consistent with their surface hydrophilicity indicated by the water contact angle. The present works revealed the attachment and growth behavior of HaCat mainly depend on the content of the membrane, not the contact angle of the membrane.

\subsection{Proliferation of HaCat Cells in the Composite Scaffolds}

$\mathrm{HaCat}$ cells were cultured in the sponge scaffolds for 1,3 , $5,7 \mathrm{~d}$, and cell proliferation was determined by MTT method. The result of MTT test is shown in Figure 10. It was clear that HaCat cells proliferated well on the scaffolds 3 and 4. On the other hand, the increasing ADM of

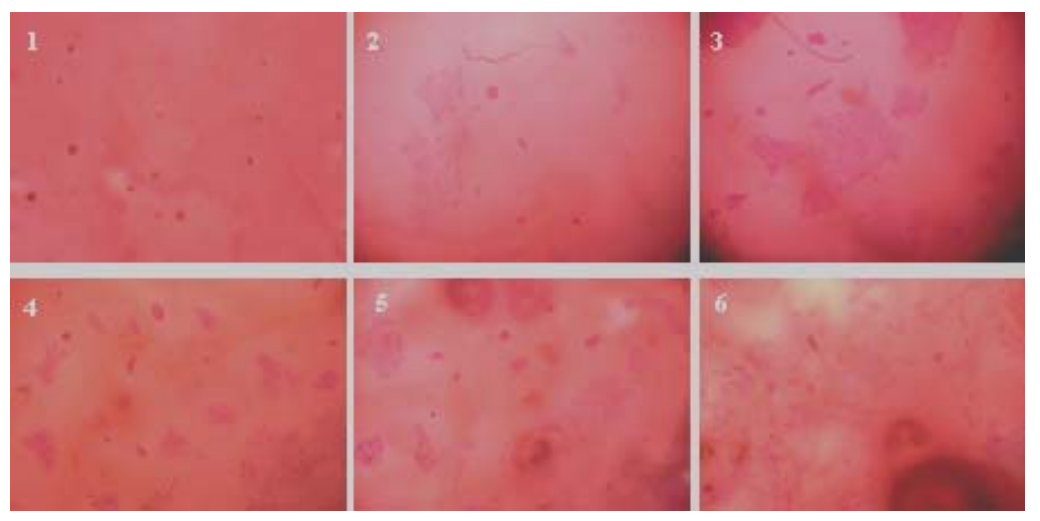

Figure 8. H.E Staining images of HaCat on composite membranes on day 5.

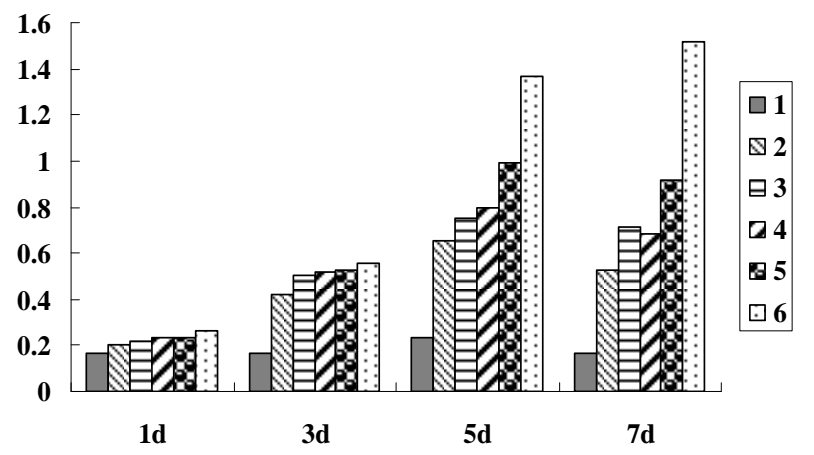

Figure 9. Proliferation of HaCat cells on the surface of composite membrane.

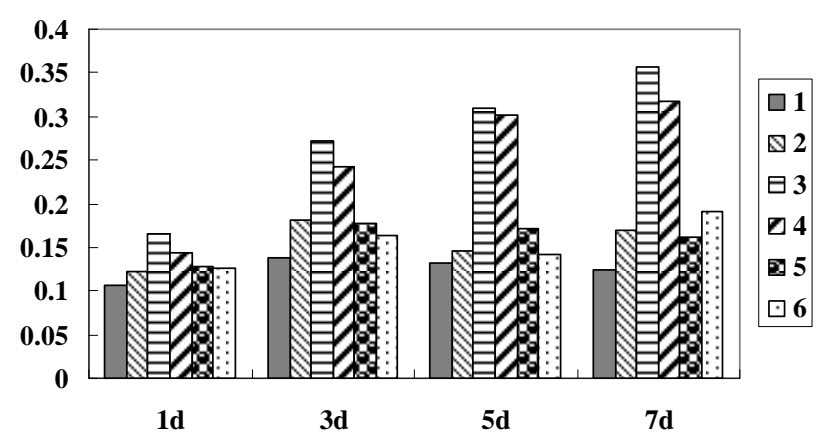

Figure 10. Proliferation of HaCat cells in the composite scaffolds. 
sample 5 and 6 scaffolds resulted in lower numbers of $\mathrm{HaCat}$ cells. This trend was not consistent with the statistical results of cell proliferation on membranes. The reason was mainly that the cell growth depended on both material content and structure of the sponge scaffolds. Control of scaffold pore morphology is critical for controlling cellular colonization rates within cell scaffold co-culture in vitro.

\section{Conclusions}

In this work, six composite membranes were prepared by solvent evaporation technique and six composite scaffolds were prepared by freeze-drying method. The effect of ADM on the characteristics of composite membranes and scaffolds were investigated by static water contact angle measurements and water absorption. The water absorption value of all scaffolds was over 18 times of its initial weight, which is high enough for skin regeneration scaffold, but there were no significant differences of water absorption ratio between deionized water and PBS solution for same scaffold $(P>0.05)$. The scaffolds morphology were observed by SEM, and the results showed that CS/ADM three-dimensional (3D) microporous structures were successfully produced. Cell compatibility of the composite membranes and scaffolds were analyzed by HaCat cells co-culture in vitro. The cell compatibility results revealed that composite membranes with higher levels of ADM content provided much better substrates in terms of cell attachment, growth and morphology. But in the scaffolds, the cell proliferation was determined not only by the hydrophilcity and content, but also by the structure of the scaffolds. As a consequence, a composite material includes membrane 6 and scaffolds 4 will be the best materilas for HaCat prolifferation. This study was the basic work for further investigation, a new human epidermal model composite material includes membrane and sponge scaffold will be reconstructed and the effect of 12 reference chemical substances in OECD TG 43 on the HaCat viability in composite material will be investigated in our future research.

\section{Acknowledgements}

The research was supported by Key Project of AMMS Innovation Fund (2008ZD09) and General Program of Natural Science Foundation of Tianjin (07JCYBJC05100).

\section{REFERENCES}

[1] K. Helena, L. Manfred and S. Horst, "Assessment of the Human Epidermis Model Skin Ethic RHE for in Vitro Skin Corrosion Testing of Chemicals According to New OECD TG 431," Toxicology in Vitro, Vol. 20, 2006, pp.
547-559. doi:10.1016/j.tiv.2005.11.008

[2] J. Hoffmann, E. Heisler and S. Karpinski, "Epidermalskin-test 1000 (EST-1000)—A new Reconstructed Epidermis for in Vitro Skin Corrosivity Testing," Toxicology in Vitro, Vol. 19, 2005, pp. 925-929. doi:10.1016/j.tiv.2005.06.010

[3] Organisation for Economic Co-operation and Development, "Acute Dermal Irritation/Corrosion," OECD Guideline for the testing of Chemicals, No. 404, Organisation for Economic Cooperation and Development, Paris, France, 2002.

[4] Organisation for Economic Co-operation and Development, "In Vitro Skin Corrosion: Human Skin Model Test," OECD guideline for testing of chemicals, No. 431, Organisation for Economic Co-operation and Development, Paris, France, 2004.

[5] J. K. F. Suh and H. W. T. Matthew, "Application of Chitosan-Based Polysaccharide Biomaterials in Cartilage Tissue Engineering: A Review," Biomaterials, Vol. 21, 2000, pp. 2589-2598. doi:10.1016/S0142-9612(00)00126-5

[6] E.B. Denkbaş and M. Odabaşi, "Chitosan Microspheres and Sponges: Preparation and Characterization," Journal of Applied Polymer Science, Vol. 76, 2000, pp. 16371643.doi:10.1002/(SICI)1097-4628(20000613)76:11<163 7::AID-APP4>3.0.CO;2-Q

[7] K. G. H. Desai and H. J. Park, "Preparation and Characterization of Drug-Loaded Chitosan-Tripolyphosphate Microspheres by Spray Drying," Drug Development Research, Vol. 64, 2005, pp. 114-128. doi:10.1002/ddr.10416

[8] Iyabo Adekogbe and Amyl Ghanem, "Fabrication and Characterization of DTBP-Crosslinked Chitosan Scaffolds for Skin Tissue Engineering," Biomaterials, Vol.26, 2005, pp. 7241- 7250 .

doi:10.1016/j.biomaterials.2005.05.043

[9] P. J. Vandevord, H. W. T. Matthew, S. P. Desilva, 1. Mayton, B. Wu and P. H. Wooley, "Evaluation of the Biocompatibility of a Chitosan Scaffold in Mice," Journal of Biomedical Materials Research, 2002, Vol. 59, pp. 585-90. doi:10.1002/jbm. 1270

[10] L. Ma, C.Y. Gao, Z. Mao, J. Zhou, J. Shen and X. Hu, "Han C: Collagen/Chitosan Porous Scaffolds with Improved Biostability for Skin Tissue Engineering," Biomaterials, Vol. 24, 2003, pp. 4833-4841. doi:10.1016/S0142-9612(03)00374-0

[11] A. N. L. Rocha, T. N. C. Dantas, J. L. C. Fonseca and M. R. Pereira, "Permeation of Drugs in Chitosan Membranes," Journal of Applied Polymer Science, Vol. 84, 2002, pp. 44-49. doi:10.1002/app.10185

[12] H. Ueno, H. Yamada, I. Tanaka and N. Kaba, Matsuura M, Okumura M, Kadosawa T and T. Fujinaga, "Accelerating Effects of Chitosan for Healing at Early Phase of Experimental Open Wound in Dogs," Biomaterials, Vol. 20, 1999, pp. 1407-1414. doi:10.1016/S0142-9612(99)00046-0

[13] C. Xiao, S. Gao, H. Wang and L. Zhang, "Blend Films from Chitosan and Konjac Glucomannan Solutions," Journal of Applied Polymer Science, Vol. 76, 2000, pp. 509-515. 
doi:10.1002/(SICI)1097-4628(20000425)76:4<509::AIDAPP8>3.0.CO;2-2

[14] T. W. Chung, Y. F. Lu, S. S. Wang, Y. S. Lin and S. H. $\mathrm{Chu}$, "Growth of Human Endothelial Cells on Photochemically Grafted Gly-Arg-Gly-Asp (GRGD) Chitosans," Biomaterials, Vol. 23, 2002, pp. 4803-4809. doi:10.1016/S0142-9612(02)00231-4

[15] Park Y. J., Lee M. Y., Lee J. Y., Seol Y. J., Chung C. P. and Lee S. J., "Controlled Release of Platelet-Derived Growthfactor-BB from Chondroitin Sulfate-Chitosan Sponge for Guided Bone Regeneration," Journal of Controlled Release, Vol. 67, 2000, pp. 385-394. doi:10.1016/S0168-3659(00)00232-7

[16] T. Suzuki, Y. Mizushima, T. Umeda and R. Ohashi, "Further Biocompatibility Testing of Silica-Chitosan Complex Membrane in the Production of Tissue Plasminogen Activator by Epithelial and Fibroblast Cells," Journal of Bioscience and Bioengineering, 1999, Vol. 88: pp. 194-199. doi:10.1016/S1389-1723(99)80201-1

[17] I. Yamaguchi, S. Itoh, M. Suzuki, M. Sakane, A. Osaka and J. Tanaka, "The Chitosan Prepared from Crab Tendon I: The Characterization and the Mechanical Properties," Biomaterials, Vol. 24, 2003, pp. 2031-2036. doi:10.1016/S0142-9612(02)00633-6

[18] J. Y. Lee, S. H. Nam, S. Y. Im, Y. J. Park, Y. M. Lee, Y. J. Seol, C. P. Chung and S. J. Lee, "Enhanced Bone Formation by Controlled Growth Factor Delivery from ChitosanBased Biomaterials," Journal of Controlled Release, Vol. 78, 2002, pp. 187-197. doi:10.1016/S0168-3659(01)00498-9

[19] S. F. Badylak, F. Ling and R. Record and J. Hodde, "Vascularization of 3-Dimensional Scaffolds. Symposium on Tissue Engineering Science," Aegean Conferences Series, Vol. 4, 2002, p. 63.

[20] P. Boukamp, R. T. Petrussevska, J. Hornung, A. Markham and N.E. Fusenig, "Normal Keratinization in a Spontaneously Immortalised Aneuploid Human Keratinocyte cell Line," The Journal of Cell Biology, Vol. 106, 1988, pp. 761-771. doi:10.1083/jcb.106.3.761

[21] V. Sundararajan, Madihally and Howard W.T. Matthew. "Porous Chitosan Scaffolds for Tissue Engineering," Biomaterials, Vol. 20, 1999, pp. 1133-1142.

[22] J. Ma, H. Wang, B. He and J. Chen, "A Preliminary in Vitro Study on the Fabrication and Tissue Engineering Applications of a Novel Chitosan Bilayer Material as a Scaffold of Human Neofetal Dermal Fibroblasts," Biomaterials, Vol. 22, 2001, pp. 331-336. doi:10.1016/S0142-9612(00)00188-5

[23] E. Behravesh, M. D. Timmer, J. J. Lemoine, M. A. K. Liebschner and A.G. Mikos, "Evaluation of the in Vitro
Degradation of Macroporous Hydrogels Using Gravimeter, Confined Compression Testing and Microcomputed Tomography," Biomacromolecules, Vol. 3, 2002, pp. 1263-1270. doi:10.1021/bm020067+

[24] J. R. Karel Smetana, Jaromir Lukas, Vera Paleckova and et al., "Effect of Ehemical Structure of Hydrogels on the Adhesion and Phenotypic Characteristics of Human Monocytes Such as Expression of Galectins and Other Carbohydrate-Binding Sites," Biomaterials, Vol. 18, 1997, pp. 1009-1014. doi:10.1016/S0142-9612(97)00037-9

[25] E. T. den Braber, J. E. de Ruijter, L. A. Ginsel and et al., "Quantitative Analysis of Fibroblast Morphology on Microgrooved Surfaces with Various Groove and Ridge Dimensions," Biomaterials, Vol. 17, 1996, pp. 2037-2044. doi:10.1016/0142-9612(96)00032-4

[26] R. Singhvi, G. Stephanopoulos and I. C. Daniel, "Effect of Substrate Morphology on Cell Physiology," Biotechnology and Bioengineering, Vol. 43, 1994, pp. 764-771. doi:10.1002/bit.260430811

[27] Takahiro Suzuki and Yasuyuki mizushima, "Characteristics of Silica-Chitosan Complex Membrane and Their Relationships to the Characteristics of Growth and Adhesiveness of L-929 Cells Cultured on the Biomembrane," Journal of Fermentation and Bioengineering, Vol. 84, 1997, pp. 128-132. doi:10.1016/S0922-338X(97)82541-X

[28] N. J. Hallab, K. J. Bundy, K. O’Connor and et al., “Cell Adhesion to Biomaterials: Correlations between Surface Charge, Surface Roughness, Adsorbed Protein and Cell Morphology," Journal of Long-Term Effects of Medical Implants, Vol. 5, 1995, pp. 209-231.

[29] T.G. Ruardy, H.E. Moorlag, J.M. Schakenraad and et al., "Growth of Fibroblasts and Endothelial Cells on Wettability Gradient Surfaces," Journal of Colloid and Interface Science, Vol. 188, 1997, pp. 209-217. doi:10.1006/jcis.1997.4769

[30] C. S. Chen, M. Mrksich, S. Huang, G. M. Whitesides and D. E. Ingber, "Geometric Control of Cell Life and Death," Science, Vol. 276, 1997, pp. 1425-1428. doi:10.1126/science.276.5317.1425

[31] E. A. Vogler and R. W. Bussian, "Short-Term Cell-Attachment Rates: A Surface-Sensitive Test of Cell-Substrate Compatibility." Journal of Biomedical Materials Research, Vol. 21, 1987, pp. 1197-1211. doi:10.1002/jbm.820211004

[32] Y. Zhu, C. Gao, T. He, X. Liu and J. Shen, "Layer-byLayer Assembly to Modify Poly(L-Lactic Acid) Surface toward Improving Its Cytocompatibility to Human Endothelial Cells." Biomacromolecules, Vol. 4, 2003, pp. 446452. doi: $10.1021 / \mathrm{bm} 025723 \mathrm{k}$ 